Modeling, Identification and Control, Vol. 32, No. 1, 2011, pp. 35-45, ISSN 1890-1328

\title{
Analysis, Modeling and Simulation of Mechatronic Systems using the Bond Graph Method
}

\author{
A. Alabakhshizadeh ${ }^{1}$ Y. Iskandarani ${ }^{2}$ G. Hovland ${ }^{2}$ O. M. Midtgård ${ }^{1}$ \\ ${ }^{1}$ Renewable Energy Group, Faculty of Engineering and Science, University of Agder, N-4898 Grimstad, Norway. \\ E-mail: \{abozar.alabakhshizadeh, ole-morten.midtgard\}@uia.no \\ ${ }^{2}$ Mechatronics Group, Faculty of Engineering and Science, University of Agder, N-4898 Grimstad, Norway. E-mail: \\ \{yousef.iskandarani, geir.hovland\} @uia.no
}

\begin{abstract}
The Bond Graph is the proper choice of physical system used for: (i) Modeling which can be applied to systems combining multidisciplinary energy domains, (ii) Analysis to provide a great value proposition for finding the algebraic loops within the system enabling the process of troubleshooting and eliminating the defects by using the proper component(s) to fix the causality conflict even without being acquainted in the proper system, and (iii) Simulation facilitated through derived state space equations from the Bond Graph model is solved using industrial simulation software, such as 20-Sim, www.20sim.com.

The Bond Graph technique is a graphical language of modeling, in which component energy ports are connected by bonds that specify the transfer of energy between system components. Following a brief introduction of the Bond Graph methodology and techniques, two separate case studies are comprehensively addressed. The first case study is a systematic implementation of a fourth order electrical system and conversion to mechanical system while the second case study presents modeling of the Dielectric Electro Active Polymer (DEAP) actuator. Building the systematic Bond Graph of multifaceted system and ease of switching between different domains are aims of the first case study while the second study shows how a complex mechatronic system could be analyzed and built by the Bond Graph. The respective Bond Graphs in each case is evaluated in the light of mathematical equations and simulations. Excellent correlation has been achieved between the simulation results and proper system equations.
\end{abstract}

Keywords: 20-Sim tool, Bond graph, Casual stroke, Dielectric electro active polymers (DEAP), Push actuator

\section{Introduction}

The Bond Graph is an abstract representation of a system where a collection of components interact with each other through energy ports and are placed in the system where energy is exchanged Wong and Rad (1998). The Bond Graph is a graphical method to modeling and simulation of multi-domain dynamic systems, in which component energy ports are connected by bonds that specify the transfer of energy between system components. Power, the rate of energy trans- port between components, is the universal currency of physical system Gawthrop and Bevan (2007).

The Bond Graph may be used to model energy transformation across many energy domains including electrical, mechanical (translation and rotation), hydraulic, thermal, magnetics and chemical. The Bond Graph energy domains for different disciplines are shown in Table 1.

The Bond Graph takes into consideration both topological and computational structure of multi-domain dynamic systems, while most other graphical tech- 
Table 1: Identification of variables Pedersen and Engja (2001)

\begin{tabular}{|c|c|c|c|c|}
\hline Energy domain & Effort (e) & Flow (f) & Momentum (p) & Displacement (q) \\
\hline Electrical & Voltage $[\mathrm{V}]$ & Current $[\mathrm{A}]$ & Flux linkage [Vs] & Charge $[\mathrm{C}]$ \\
\hline $\begin{array}{l}\text { Mechanical } \\
\text { translation }\end{array}$ & Force $[\mathrm{N}]$ & Velocity $[\mathrm{m} / \mathrm{s}]$ & $\begin{array}{c}\text { Linear momentum } \\
{[\mathrm{kgm} / \mathrm{s}]}\end{array}$ & Distance $[\mathrm{m}]$ \\
\hline $\begin{array}{l}\text { Mechanical } \\
\text { rotation }\end{array}$ & Torque $[\mathrm{Nm}]$ & $\begin{array}{l}\text { Angular velocity } \\
{[\mathrm{rad} / \mathrm{s}]}\end{array}$ & $\begin{array}{c}\text { Angular } \\
\text { momentum }[\mathrm{Nms}]\end{array}$ & Angle [rad] \\
\hline Hydraulic & Pressure $[\mathrm{Pa}]$ & $\begin{array}{l}\text { Volume flow rate } \\
\qquad\left[\mathrm{m}^{3} / \mathrm{s}\right]\end{array}$ & $\begin{array}{l}\text { Pressure } \\
\text { momentum } \\
{\left[N / m^{2} s\right]}\end{array}$ & Volume $\left[\mathrm{m}^{3}\right]$ \\
\hline Thermal & Temperature $[\mathrm{K}]$ & Entropy flow $[\mathrm{J} / \mathrm{s}]$ & - & Entropy $[\mathrm{J}]$ \\
\hline Magnetic & $\begin{array}{l}\text { Magneto motive } \\
\text { force }[\mathrm{A}]\end{array}$ & Flux rate $[\mathrm{Wb} / \mathrm{s}]$ & - & Flux $[\mathrm{Wb}]$ \\
\hline Chemical & $\begin{array}{c}\text { Chemical potential } \\
{[\mathrm{J} / \mathrm{mol}]}\end{array}$ & $\begin{array}{l}\text { Rate of reaction } \\
{[\mathrm{mol} / \mathrm{s}]}\end{array}$ & - & $\begin{array}{l}\text { Advancement of } \\
\text { Reaction [mol] }\end{array}$ \\
\hline
\end{tabular}

niques preserve only topological or computational structure. For instance, a circuit diagram reflects the topological structure of the system while a signal flow graph and block diagram are used for computational structure of the system. These methods are not always applicable to different multi-domain systems; e.g. a circuit diagram is used only for electrical systems.

Some of the advantages of the Bond Graph method emphasized in the literature are information about constrained states, algebraic loops, and the benefits and consequences of potential approximations and simplifications. Moreover, due to causality assignment the method gives the possibility of localization of state variables, a tool for finding and removing algebraic loops and achieving a well-behaved mathematical model. The Bond Graph provides information regarding the structural properties of the system, in terms of controllability and observability. Altogether, it is ideally suited for modeling and simulation of mechatronic systems Khurshid and Malik (2007), Roman et al. (2010).

Although the Bond Graph technique has been invented by Professor H. M. Paynter at MIT, approximately six decades ago, still it has not been one of the main tools for modeling systems. Here, in this paper, the Bond Graph technique is introduced, for the first time, to the Modeling, Identification and Control (MIC) community. After 30 years of publication, not a single paper about the Bond Graph has been published in MIC.

The paper is organized as follows: (i) Fundamentals of the Bond Graph is presented. (ii) Case study I "Fourth Order Electrical System" is introduced and analyzed using the Bond Graph and equations are extracted. Then conversion from electrical to mechanical domain is accomplished, and the results are proved with simulation software. (iii) Case study II "Dielectric Electro Active Polymer Actuator" is presented, governing equations are derived and explained. The Bond Graph model is constructed, and simulation results are shown to match well with the governing output of the equations.

\section{Fundamentals of the Bond Graph}

Components regardless of any energy domains are connected through a lossless line so called power bond. The direction in which the power flow is assigned, a positive value is indicated by a half-arrow on one end of the bond as it is shown in Figure 1-a. In the Bond Graph method, power consists of two variables which are known as generalized effort and generalized flow denoted by $e$ and $f$ respectively. The power flowing in the bond is defined as the product of an effort and a flow variable, as shown in Equations 1,

$$
P(t)=e(t) \times f(t)
$$

In order to describe a system, generalized momentum and generalized displacement are defined. Generalized momentum is the time integral of effort which is assigned by $p$ and generalized displacement is the time integral of flow assigned by $q$.

In the Bond Graph language, voltage and current which resemble the power variables are translated to effort and flow respectively. Furthermore, flux leakage variable and the charge are considered as momentum and displacement. Electrical elements are classified as one-port or two-port elements in the Bond Graph method according to their number of inputs or outputs. 


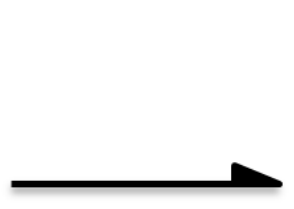

(a)
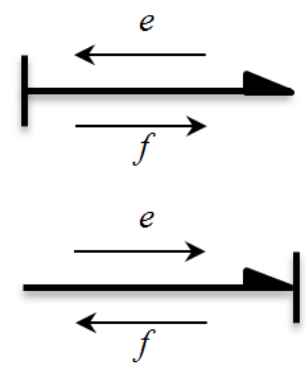

(b)
Figure 1: (a) Sign convention on the power bond. (b) Notation convention for effort and flow respect to the indicated causality on a bond

Meaning that, each bond represents two connections in the electrical system. For instance resistor, inductor, capacitor and sources are considered as one-port element while transformer is categorized as two-port element. The common electrical elements and their respective Bond Graph and constitutive relations are summarized in Table 2.

Table 2: Common electrical elements and their proper Bond Graph indicators and relations

\begin{tabular}{|c|c|c|}
\hline $\begin{array}{l}\text { Common } \\
\text { electrical } \\
\text { elements }\end{array}$ & $\begin{array}{c}\text { Bond Graph } \\
\text { element }\end{array}$ & $\begin{array}{c}\text { Constitutive } \\
\text { relations }\end{array}$ \\
\hline $\begin{array}{l}\text { Voltage } \\
\text { source }\end{array}$ & $S_{e} \stackrel{e}{\longrightarrow}$ & $e=e(t)$ \\
\hline $\begin{array}{l}\text { Current } \\
\text { source }\end{array}$ & $S_{f} \underset{f}{\longrightarrow}$ & $f=f(t)$ \\
\hline Resistor & $\frac{e}{f} R$ & $\begin{array}{c}e=\varphi_{R}(f) \\
e=R f\end{array}$ \\
\hline Inductor & $\frac{e}{f} I$ & $\begin{array}{c}p=\varphi_{I}(f) \\
p=I f\end{array}$ \\
\hline Capacitor & $\frac{e}{f} C$ & $\begin{array}{c}q=\varphi_{C}(e) \\
q=C e\end{array}$ \\
\hline Transformer & $\frac{e_{1}}{f_{1}} \mathrm{TF} \frac{e_{2}}{f_{2}}$ & $\begin{array}{l}e_{1}=e_{2} m \\
f_{1} m=f_{2}\end{array}$ \\
\hline Gyrator & $\frac{e_{1}}{f_{1}} \mathrm{GY} \frac{e_{2}}{f_{2}}$ & $\begin{array}{l}e_{1}=f_{2} r \\
f_{1} r=e_{2}\end{array}$ \\
\hline
\end{tabular}

A tetrahedron diagram which indicates the relation of the power variables; effort and flow, and energy variables; momentum and displacement for one-port elements is shown in Figure 2.

Junctions serve to interconnect other components into subsystem or system models. There are two basic junctions defined in the Bond Graph method; 0junction and 1-junction. 0 -junction which is indicated as 0 is the effort equalizing junction or flow summing junction. While 1-junction is indicated as 1 and it is flow equalizing junction or effort summing junction.

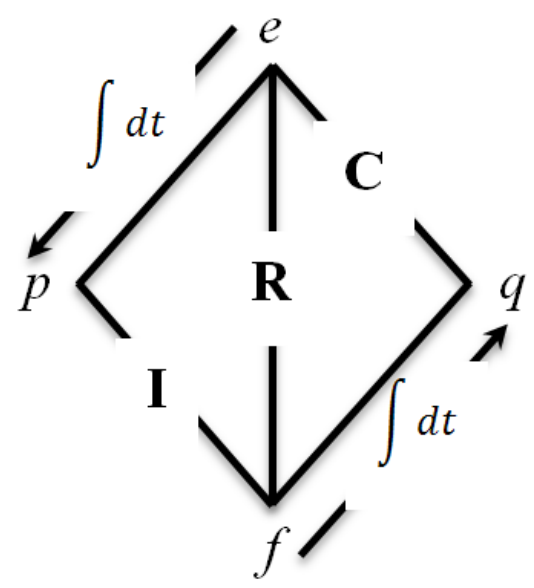

Figure 2: Tetrahedron of state for one-port elements Pedersen and Engja (2001)

In electrical domain, 0-junction is used for parallel connection which is following the Kirchhoff's voltage law. On the other hand, 1-junction restricted for series connection which is following the Kirchhoff's current law.

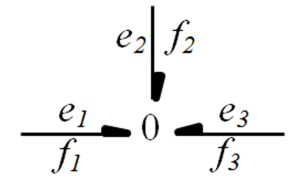

(a)

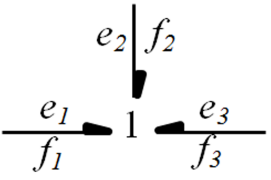

(b)
Figure 3: The two basic junctions. (a) 0-junction and (b) 1-junction

The constitutive relations for 0 -junction and 1junction are summarized in Equations 2 and 3 as follows;

$$
\begin{aligned}
& 0-\text { Junction }:\left\{\begin{array}{c}
e_{1}=e_{2}=e_{3} \\
f_{1}+f_{2}+f_{3}=0
\end{array}\right. \\
& 1-\text { Junction }:\left\{\begin{array}{c}
f_{1}=f_{2}=f_{3} \\
e_{1}+e_{2}+e_{3}=0
\end{array}\right.
\end{aligned}
$$

Causality is perhaps the most significant concept embedded in the Bond Graph in order to extract the state space equations. Causality is used to define which energy variables are input variables and which are output variables with respect to elements considered and represented by causal stroke, placed perpendicular to the 
bond at one of its ends. The causal stroke indicates the direction of the effort and flow. The direction of the causal stroke is independent of the power direction, which is shown in Figure 1-b.

The possible causalities and their respective relations for each electrical element are indicated in Table 3. According to Table 3, both voltage and current sources have a constant causality while the rest of the elements could vary between integral and derivative causality. However, the most desirable causality of the storage elements is the integral causality.

Table 3: Common electrical elements and their proper Bond Graph indicators and causal relations

\begin{tabular}{|c|c|c|}
\hline $\begin{array}{l}\text { Common } \\
\text { electrical } \\
\text { elements }\end{array}$ & $\begin{array}{c}\text { Bond } \\
\text { Graph } \\
\text { element }\end{array}$ & $\begin{array}{l}\text { Causal } \\
\text { relations }\end{array}$ \\
\hline $\begin{array}{l}\text { Voltage } \\
\text { source }\end{array}$ & $S_{e} \rightarrow$ & $e(t)=$ given \\
\hline $\begin{array}{l}\text { Current } \\
\text { source }\end{array}$ & $S_{f} \mapsto$ & $f(t)=$ given \\
\hline Resistor & $\begin{array}{l}\mapsto R \\
\dashv R\end{array}$ & $\begin{array}{c}e=\varphi_{R}(f) \\
f=\varphi_{R(e)}^{-1}\end{array}$ \\
\hline Inductor & $\begin{array}{l}\mapsto I \\
\rightarrow I\end{array}$ & $\begin{array}{c}e=\frac{d}{d t}\left[\varphi_{I}(f)\right] \\
f=\varphi_{I}^{-1}\left(\int e d t\right)\end{array}$ \\
\hline Capacitor & $\begin{array}{l}\mapsto C \\
\rightarrow C\end{array}$ & $\begin{array}{c}e=\varphi_{C}^{-1}\left(\int f d t\right) \\
f=\frac{d}{d t}\left[\varphi_{C}(e)\right]\end{array}$ \\
\hline Transformer & $\begin{array}{l}\mapsto \mathrm{TF} \mapsto \\
\mapsto \mathrm{TF} \rightarrow\end{array}$ & $\begin{array}{c}e_{1}=m e_{2}, f_{2}=m f_{1} \\
e_{2}=\frac{e_{1}}{m}, f_{1}=\frac{f_{2}}{m} \\
e_{1}=r f_{2}\end{array}$ \\
\hline Gyrator & $\begin{array}{l}\mapsto \mathrm{GY}-1 \\
-\mathrm{GY} \mapsto\end{array}$ & $\begin{aligned} e_{2} & =r f_{1} \\
f_{1} & =\left(\frac{1}{r}\right) e_{2} \\
f_{2} & =\left(\frac{1}{r}\right) e_{1}\end{aligned}$ \\
\hline
\end{tabular}

\section{Case Study I}

\subsection{Electrical Model and Reduced Bond Graph Model}

A fourth order electrical circuit is considered as a case study as shown in Figure 4. It consists of a common voltage source and a resistor that connects to two parallel branches containing two inductors and two capacitors which are connected diagonally.

Following the step-by-step procedure to achieve a Bond Graph of electrical circuits can become an issue for complicated circuits. The algorithmic Bond Graph technique for electrical circuits is the solution when challenging circuits exist. In the algorithmic Bond Graph technique when it is applied to electric current, first, the points with different voltages are identified

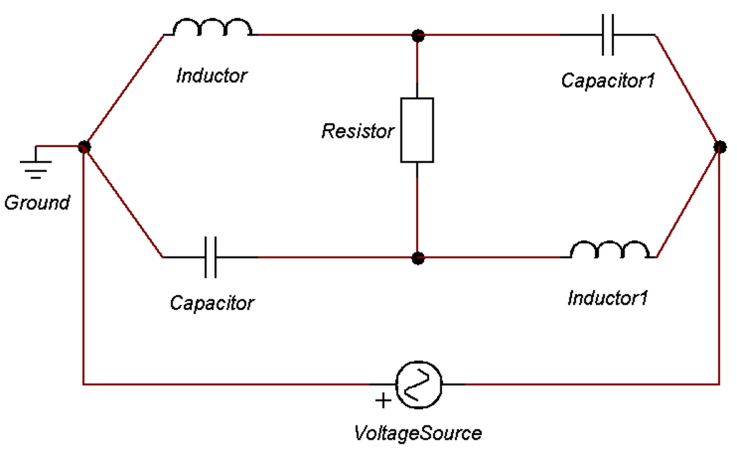

Figure 4: A forth order electrical circuit

and each indicated as a 0 -junction. Meaning that, each element is located between two 0-junctions. Second, the proper element is connected to the 1-junction, connected to the related 0 -junctions. Third, defining the ground junction by connecting the corresponding 0 -junction to the zero voltage source $\left(S_{e}=0\right)$ element. Forth, simplifying the Bond Graph by neglecting the 0 -junction which is connected to the ground and obtaining the reduced Bond Graph.

The algorithmic Bond Graph technique for the selected electrical circuit is indicated in Figure 5-a to c.

\subsection{Bond Graph Model Equations}

To obtain the state space equations for a reduced Bond Graph, first the flow equations in 0-junctions and effort equations in 1-junctions are attained as shown in equations (4-9), respectively;

$$
\begin{gathered}
f_{1}=f_{12}+f_{2} \\
f_{5}=f_{4}-f_{6} \\
f_{10}=f_{9}+f_{8} \\
e_{3}=e_{2}-e_{4} \\
e_{7}=e_{6}+e_{8} \\
e_{10}=e_{12}-e_{11}
\end{gathered}
$$

Equations (4-9) are most likely to be used in second and third step of acquiring the model equations.

Second, the flow or effort caused by each element is obtained. It is recommended to start with sources (e.g. voltage and current source), then the storage elements (e.g. I and C), and finally non-storage elements 


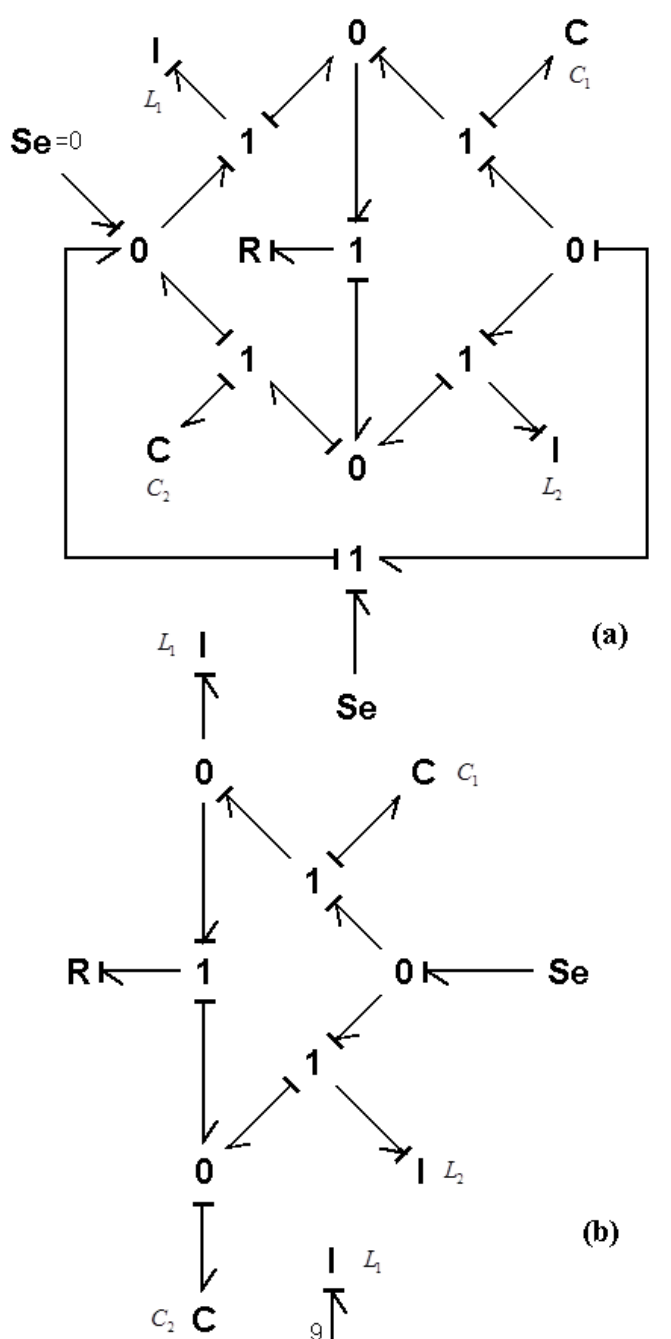

(e.g. R). Equations are derived and shown in (10-15) as follows;

$$
\begin{gathered}
S_{e}: e_{1}=E(t) \\
L_{1}: f_{9}=\frac{p_{9}}{L_{1}} \\
L_{2}: f_{3}=\frac{p_{3}}{L_{2}} \\
C_{1}: e_{11}=\frac{q_{11}}{c_{1}} \\
C_{2}: e_{5}=\frac{q_{5}}{c_{2}}
\end{gathered}
$$

$$
\begin{aligned}
R & : f_{7}=\frac{e_{7}}{R}=\frac{1}{R}\left(e_{6}+e_{8}\right)=\frac{1}{R}\left(e_{5}+e_{12}-e_{11}\right) \\
& =\frac{1}{R}\left(e_{5}+e_{1}-e_{11}\right)=\frac{q_{5}}{R C_{2}}+\frac{E(t)}{R}-\frac{q_{11}}{R C_{1}}
\end{aligned}
$$

Third, the received flow or effort of each storage element with integral causality from the system should be obtained which are the state space equations; shown in the equations (16-19).

$$
\begin{gathered}
\begin{aligned}
L_{1}: e_{9}=\dot{p}_{9} \rightarrow \dot{p}_{9}= & e_{10}=e_{12}-e_{11} \\
& =e_{1}-e_{11}=E(t)-\frac{q_{11}}{C_{1}}
\end{aligned} \\
\begin{aligned}
L_{2}: e_{3}=\dot{p}_{3} \rightarrow \dot{p}_{3}= & e_{2}-e_{4} \\
& =e_{1}-e_{5}=E(t)-\frac{q_{5}}{C_{2}}
\end{aligned} \\
C_{1}: f_{11}=\dot{q}_{11} \rightarrow \dot{q}_{11}=f_{10}=f_{9}+f_{8}=f_{9}+f_{7} \\
=\frac{p_{9}}{L_{1}}+\frac{q_{5}}{R C_{2}}+\frac{E(t)}{R}-\frac{q_{11}}{R C_{1}} \\
C_{2}: f_{5}=\dot{q}_{5} \rightarrow \dot{q}_{5}=f_{4}-f_{6}=f_{3}-f_{7} \\
=\frac{p_{3}}{L_{2}}-\frac{q_{5}}{R C_{2}}-\frac{E(t)}{R}+\frac{q_{11}}{R C_{1}}
\end{gathered}
$$

Finally, the state space equations are summarized in the matrix below.

$$
\left\{\begin{array}{l}
\dot{x}(t)=A(t) x(t)+B(t) u(t) \\
y(t)=C(t) x(t)+D(t) u(t)
\end{array}\right.
$$




$$
\begin{gathered}
{\left[\begin{array}{c}
\dot{p}_{9} \\
\dot{p}_{3} \\
\dot{q}_{11} \\
\dot{q}_{5}
\end{array}\right]=\left[\begin{array}{cccc}
0 & 0 & -\frac{1}{C_{1}} & 0 \\
0 & 0 & 0 & -\frac{1}{C_{2}} \\
\frac{1}{L_{1}} & 0 & -\frac{1}{R C_{1}} & \frac{1}{R C_{2}} \\
0 & \frac{1}{L_{2}} & \frac{1}{R C_{1}} & -\frac{1}{R C_{2}}
\end{array}\right] \cdot\left[\begin{array}{c}
p_{9} \\
p_{3} \\
q_{11} \\
q_{5}
\end{array}\right]} \\
+\left[\begin{array}{c}
1 \\
1 \\
\frac{1}{R} \\
-\frac{1}{R}
\end{array}\right] E(t) \quad(21) \\
e_{7}=\left[\begin{array}{llll}
0 & 0 & -\frac{1}{C_{1}} & -\frac{1}{C_{2}}
\end{array}\right] \cdot\left[\begin{array}{c}
p_{9} \\
p_{3} \\
q_{11} \\
q_{5}
\end{array}\right]+E(t)
\end{gathered}
$$

\subsection{Electrical to Mechanical System Conversion}

As mentioned, the main value proposition of the Bond Graph technique is its multi-domain characteristics. In order to have equivalent systems in different domains, first the Bond Graph should be sketched. Having the Bond Graph, it is easy to switch between domains.

In the mechanical domain, damper, mass, and spring are equivalent to $\mathrm{R}$, I, and $\mathrm{C}$ respectively. Furthermore, sources of current and voltage in electrical domain equate to sources of velocity and force in the mechanical domain and represented by $S_{f}$ and $S_{e}$ respectively. 1-junctions in the Bond Graph, indicate the equal flow and each 0-junction connected to the elements indicates there is a mechanical element between the associated 1-junctions.

In Figure 5-c, three 1-junctions are translated to three separate equal flows. Thus, two masses instead of $L_{1}$ and $L_{2}$ and two springs instead of $C_{1}$ and $C_{2}$ appear in the mechanical system. A source of effort and a damper are replaced with the $S_{e}$ and R. Figure 6 presents the mechanical system of the Bond Graph sketched in Figure 5-c which is equivalent to the electrical system argued as the case study, Figure 4.

\subsection{Simulation Results}

Several computer-based simulation tools are available for designing and simulating Bond Graphs. 20-Sim is a graphical modeling and simulation program which is suitable for generating and processing of dynamic systems, such as electrical, mechanical, and hydraulic systems or any combination of these in an intuitive and user-friendly way. The software has been used for simulating the three approaches simultaneously; Electrical system, Bond Graph and mechanical system. Table 4 shows the values of each parameter.

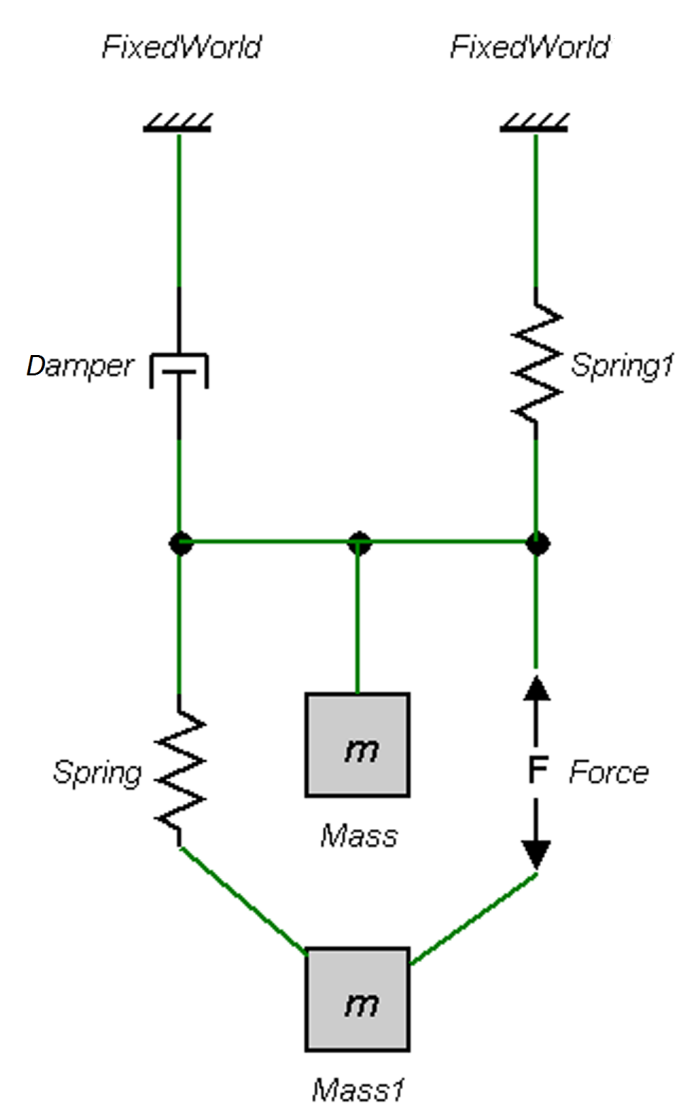

Figure 6: Mechanical model of the Bond Graph and electrical model shown in Figure 5-c and Fig-

\begin{tabular}{|c|c|c|c|}
\hline Value & $\begin{array}{l}\text { Bond } \\
\text { Graph }\end{array}$ & $\begin{array}{c}\text { Electrical } \\
\text { Domain }\end{array}$ & $\begin{array}{l}\text { Mechanical } \\
\text { Domain }\end{array}$ \\
\hline $200-60$ & $S_{e}$ & $\begin{array}{l}\text { Voltage } \\
\text { source } \\
\text { (V-Hz) }\end{array}$ & $\begin{array}{c}\text { Fource } \\
\text { (N-Freq) }\end{array}$ \\
\hline 0.001 & I & $\begin{array}{l}\text { Inductors } \\
(\mathrm{H})\end{array}$ & $\begin{array}{l}\text { Mass } \\
(\mathrm{Kg})\end{array}$ \\
\hline $1 \mathrm{e}-006$ & $\mathrm{C}$ & $\begin{array}{c}\text { Capacitance } \\
\text { (F) }\end{array}$ & $\begin{array}{l}\text { Spring } \\
(\mathrm{N} / \mathrm{m})\end{array}$ \\
\hline 10 & $\mathrm{R}$ & $\begin{array}{l}\text { Resistor } \\
\qquad(\Omega)\end{array}$ & $\begin{array}{l}\text { Damper } \\
\text { (N.s/m) }\end{array}$ \\
\hline
\end{tabular}
ure 4 respectively.

Table 4: Model Parameters 
Figure 7, shows the simulation results for three equivalent systems. Voltage applied to the resistor in electrical model, force applied the damper in the mechanical model, and $\mathrm{R}$ in the Bond Graph model are sketched.

The simulation results show that the obtained Bond Graph and mechanical system are equivalent with the electrical system.

\section{Case Study II}

\subsection{Dielectric Electro Active Polymer Actuator}

Danfoss PolyPower A/S has been researching into the technology of the DEAP for a number of years, using smart compliant electrode technology Benslimane et al. (2002) in conjunction with a silicon elastomer Wacker Chemie AG (2011). So far the company has concentrated on developing an automatic manufacturing facility for their PolyPower material as well as designing and fabricating PolyPower actuators. Two actuator types currently exist; a pre-strained 'pull'-type actuator and a core free tubular 'push' actuator with no pre-strain. The surface structure and the electrodes on their DEAP film are corrugated. The corrugation allow the elastomer and the electrodes to be compliant in one direction and stiff in the other direction. Applying voltage between the electrodes results in electrostatic forces and contraction between the electrodes. The resulting stress from the electro static forces causes the elastomer to elongate in one direction.

A large number of different types of DEAP actuators have been demonstrated so far. Most notable examples include planar devices, rolls, tubes, stacks, diaphragms and extenders. Of the range of DEAP-based actuators that currently exists those having a cylindrical configuration (rolls and tubes) are among the most promising and important. This kind of device was proposed for the first time by Pelrine et al. (2001), who called it a tube actuator. The basic principle of the tube actuator is that by applying a voltage to two compliant electrodes attached to the internal and external surfaces of a thin-walled cylindrical dielectric elastomer tube, the tube wall will squeeze, causing an axial elongation.

The spring roll actuator Pelrine et al. (2001), Ashley (2003) is perhaps currently the most advanced cylinder-type design for achieving large activation forces with dielectric elastomers. The actuator is comprised of a bidirectionally pre-stretched and doubleside-coated dielectric film wrapped around an elastic coil spring. The interface for the external fixing of the actuator is made from two threaded rods, which are screwed from both sides into the coil spring. In or- der to transmit the forces from the dielectric film to the spring, the rolled film is glued to the treaded rods. This actuator elongates in the axial direction when a voltage is applied and contracts back to its original length when deactivated. Elongations up to $26 \%$ and forces up to $15 \mathrm{~N}$ were achieved at $2500 \mathrm{~V}$. This design was chosen for the dielectric actuators to be used in an arm wrestling robot where 256 spring roll actuators with $12 \mathrm{~mm}$ outer radius were used.

The actuating performances of the elastomeric dielectric materials, used as electromechanical polymer transducers, have been assessed and continuously improved over the last few years so that devices made of DEAP today represent one of the best smart material technologies using polymer actuation.

This work investigates the PolyPower DEAP actuator modeling using the Bond Graph method. The Bond Graph representation of the DEAP based actuator is an alternative for the better known block diagram and signal flow Graph with a major difference of having a bidirectional physical energy exchange in between each of the Bond Graph elements. The work will provide an insight into (i) the state-of-art Bond Graph modeling of the DEAP push actuator and (ii) the simulation to show the actuator performances. The following objectives are addressed:

- An introduction to the governing equations which are used for modeling the DEAP push actuator.

- The Bond Graph model of the DEAP actuator.

- The simulations showing (i) Force-Voltage, (ii) Stroke-Voltage and (iii) Force-Stroke relations.

\subsection{Governing Equations}

Figure 8-a shows the push actuator which is produced by rolling a long laminated sheet of Electro Active Polymer 'EAP' material resulting into multi 'cylindrical' hollow tube as shown in Figure 8-b.

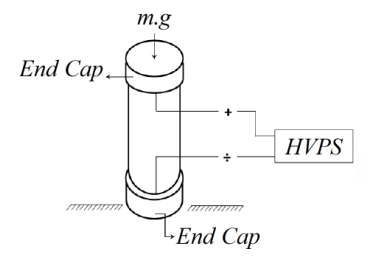

(a)

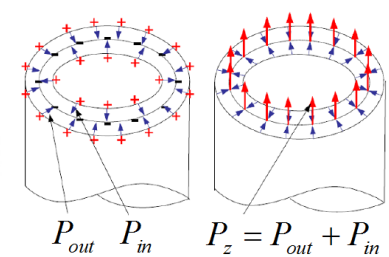

(b)
Figure 8: (a) The DEAP push actuator, (b) (i) Inner and outer pressure when electric field applied (ii) Resultant longitudinal pressure.

These actuators are envisaged to be used as hydraulic type positioning devices. To model the force 


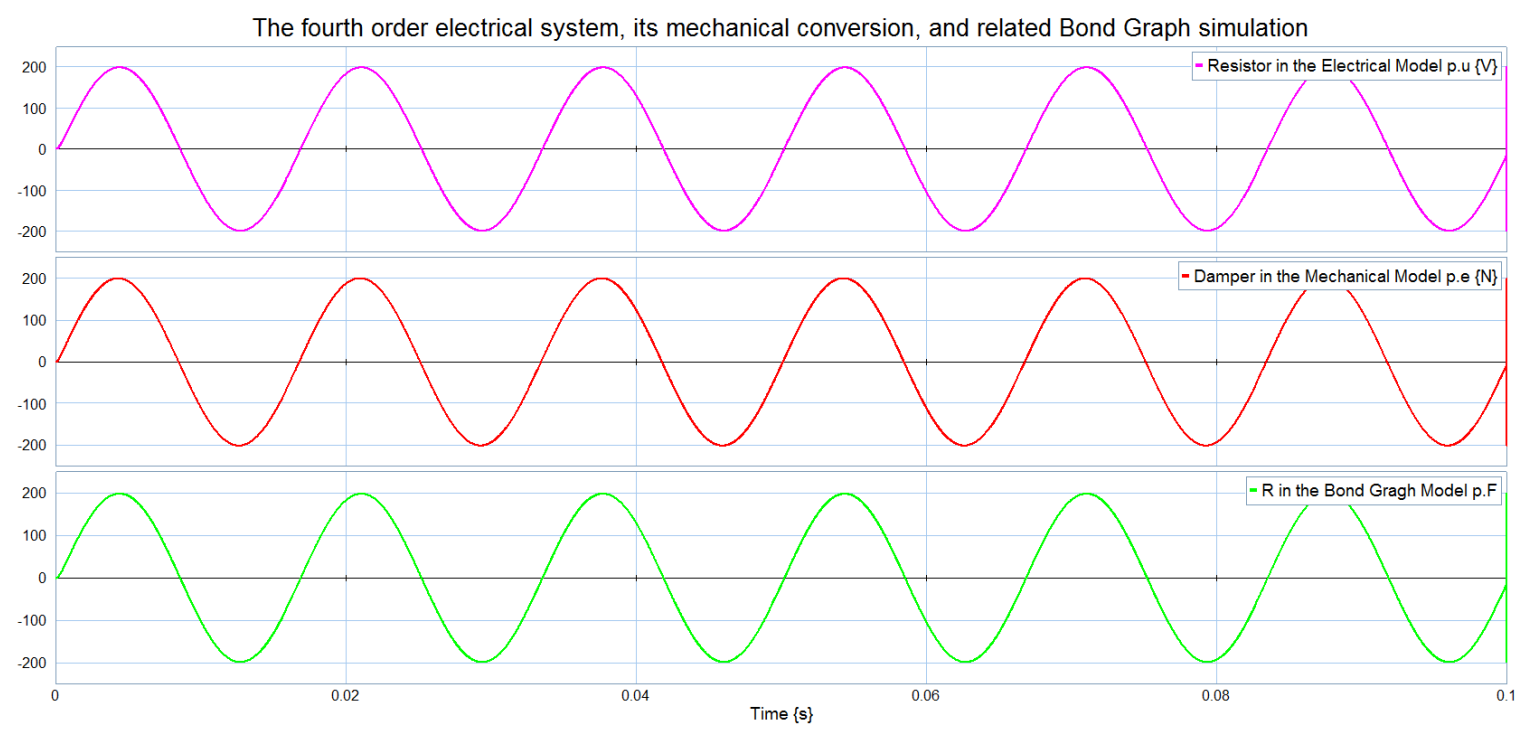

Figure 7: Simulation results: Resistor Voltage in Electrical Model (Top), Damper Force in Mechanical Model (Middle), R element effort in Bond Graph (Bottom).

characteristics of the actuator the rolled single EAP laminated sheet will be approximated as a number of concentric cylinders, within the outer and inner radii of the tubular actuator. Each 'cylinder' in the actuator contributes to the total force provided by the actuator. Since each cylinder has different geometrical dimension, each of them will contribute a different force to the overall force ' $F$ ' Iskandarani (2008) which is found to be:

$$
F a=\frac{\epsilon_{0} \cdot \epsilon_{r} \cdot y \cdot U^{2}}{z_{0}}
$$

Where $\epsilon_{0}$ and $\epsilon_{r}$ are the dielectric and relative constants, $y$; the width of the DEAP sheet, $z_{0}$; the original thickness, and $U$; is the applied voltage.

An applied electrical potential, of opposite signs, on each of the actuator's electrodes will cause the electrodes to attract each other compressing the cylinders wall thickness. The compression of the wall thickness results in elongation of the cylinder as shown in Figure 8-b. The electromechanical model of the strain ' $S$ ' of a cylindrical EAP actuator Iskandarani (2008) is found to be:

$$
S=\frac{2 \cdot \nu \cdot F a}{Y \cdot A}
$$

Where $\nu$ and $Y$ represent the Poisson's ratio and Young's modulus of the material and $A$ represents the cross sectional area for the used sheet of material. The effective stroke $\Delta L$ can be found as follows:

$$
\Delta L=S \cdot x
$$

Where $x$ is the total length of the actuator and can be found by summing active and passive length.

It is assumed that the concentric cylinders which compose the push actuator are perfectly in contact with each other such that the outer radius of one cylinder is equal to the inner radius of the next one. The inner and outer circumferences of each cylinder are force in the material when it is extended. This elastic force acts in the opposite direction to the actuator force. Since the actuator has to compress the passive part, as shown in Figure 9-a and Figure 9-b, before moving the load it looses some force.

The effective force of a cylindrical EAP actuator containing passive parts as shown by Wissler et al. (2007) is reduced as a function of the ratio of the active length to the total length as shown in the formula below.

$$
F_{e}=F_{a}\left(\frac{L_{a}}{L_{a}+L_{p}}\right)
$$

where $F_{e}$ is the effective force, $L_{a}$ and $L_{p}$ are active and the passive actuator length respectively.

\subsection{DEAP with Bond Graph}

The graphical representation of the DEAP push actuator as shown in Figure 10 has been successfully implemented using the Bond Graph. The model has been implemented and simulated using the 20-sim software. In the Bond Graph model, the actuator is divided into three different parts; two passives and one active. The active part of the actuator consists of (i) ' $C$ '; the active spring component and (ii) ' $R$ '; the active damping 


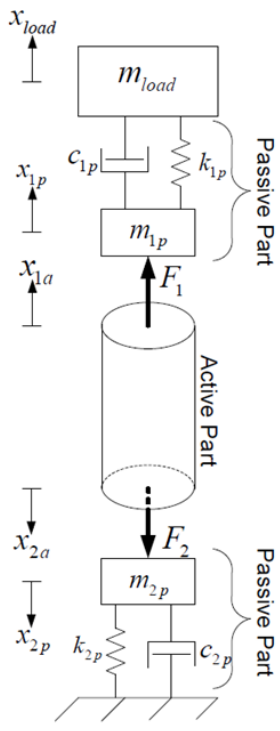

(a)

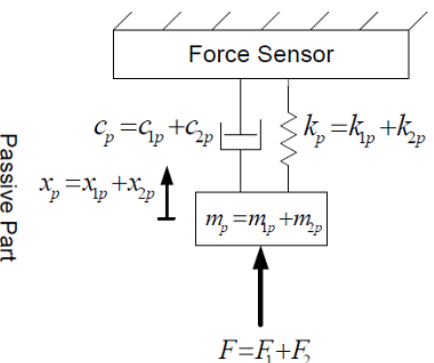

(b)

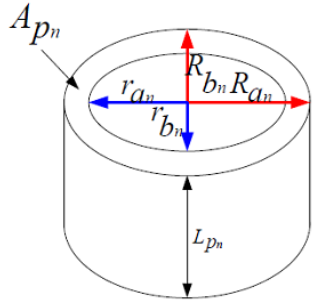

(b)
Figure 9: (a) Model of the Actuator showing the active and passive parts (b) combining top and bottom mass-spring-damper systems of passive parts.

of the actuator which shares the same flow at the ' 1 ' junction. A transformer element ' $\mathrm{TF}$ '; is used to convert the electrical power to mechanical one. Hence, it is connected to the ' 0 ' junction. The transformer provides the Maxwell Force which is generated when biasing the actuator.

The passive ends are similar, both consist of ' $\mathrm{C}, \mathrm{R}$ \& I' elements which represent the spring, damping and mass of the passive material. The ' $\mathrm{C}, \mathrm{R}$ ' elements share the same flow at the ' 1 ' junction. Hence, the ' $C, R$ ' elements of the lower passive part share the same flow with the 'I' inertial element at the ' 1 ' junction. Note that the lower passive end is fixed with a ' 0 ' junction connection to zero flow source whereby the upper passive part is loaded with an external mass which is represented by an ' $\mathrm{I}$ ' inertial element.

\subsection{Simulation Results}

In this part the 20-sim tool has been utilized to model and simulate the Bond Graph for the DEAP actuator. During the simulation phase, some parameters which characterize the push actuator properties has to be set as shown in Table 5. It is noted, the formulas which used these parameters are hidden inside the Bond Graph elements.

Based on the parameters shown in Table 5, simulations are carried out in order to determine the charac-

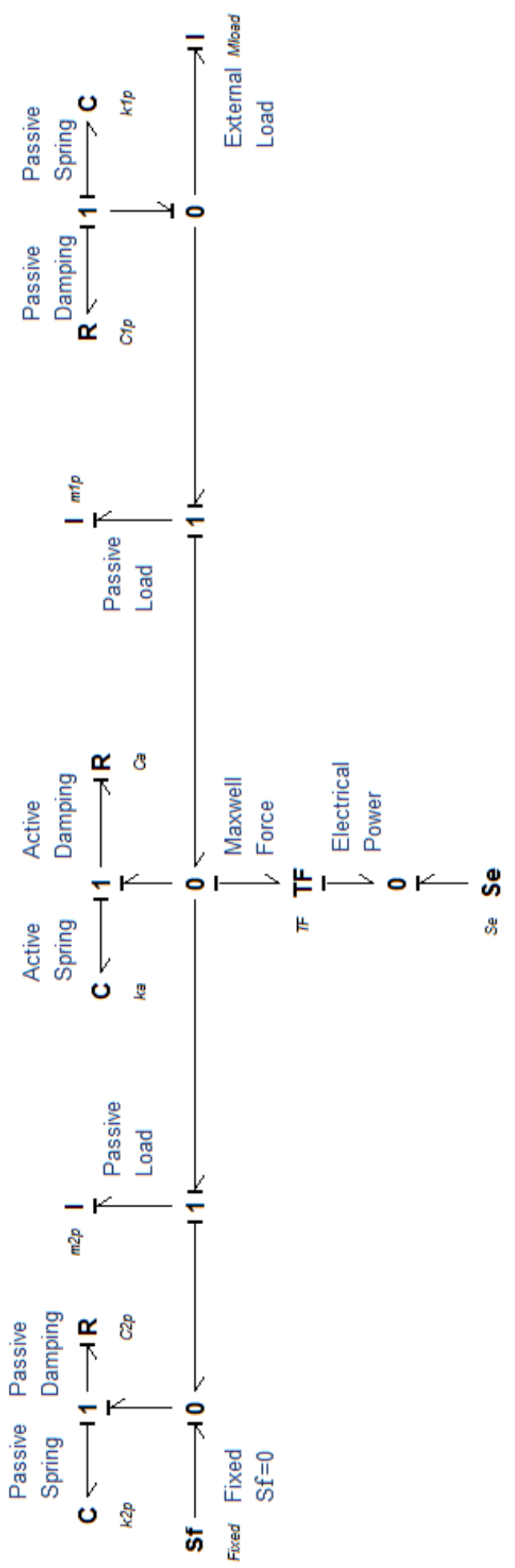

Figure 10: The DEAP push actuator model using the Bond Graph 
Table 5: The actuator parameters

\begin{tabular}{ccc}
\hline Attribute & Unit & Value \\
\hline$\epsilon_{0}$ & - & $8.8542 \mathrm{e}-12$ \\
$\epsilon_{r}$ & - & 2.733 \\
$\nu$ & - & 0.5 \\
$\mathrm{Y}$ & $\mathrm{Pa}$ & $1.925 \mathrm{e} 6$ \\
$x_{a}$ & $\mathrm{~m}$ & 0.06 \\
$x_{b}$ & $\mathrm{~m}$ & 0.04 \\
$z_{0}$ & $\mathrm{~m}$ & $75 \mathrm{e}-6$ \\
$\mathrm{y}$ & $\mathrm{m}$ & 7 \\
$\mathrm{U}$ & $\mathrm{V}$ & 3000 \\
\hline
\end{tabular}

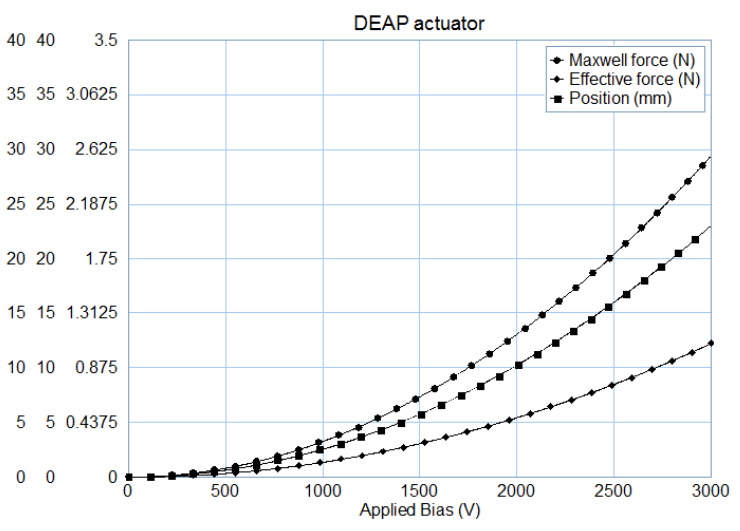

Figure 11: The actuator Maxwell and Effective Forces and Position vs. Applied voltage

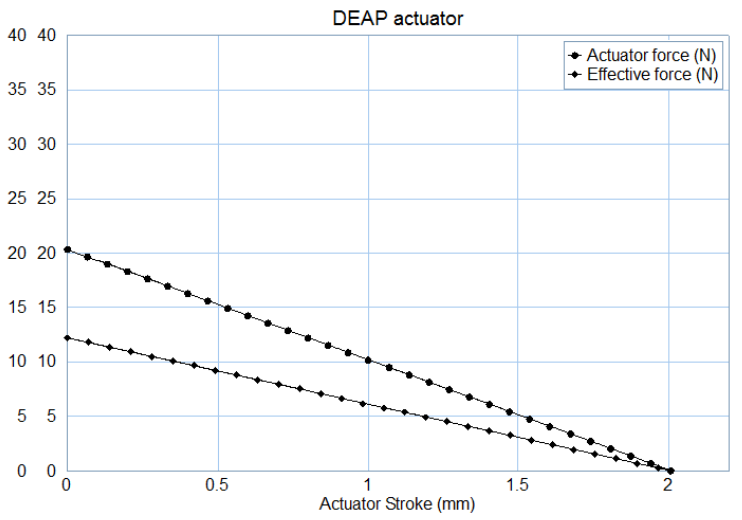

Figure 12: The push actuator Maxwell and Effective Force vs. Effective Stroke curves teristics of the DEAP push actuator. Figure 11 shows the Maxwell Force, Effective Force and Position vs. Applied Voltage curves for the given actuator properties. However, when specifying an actuator, only active length and width within limitations are specified. The parameters in Table 5 can vary, even within the same batch.

In contrast to the relations shown in Figure 11, Figure 12 shows the relation between the Effective Force and Effective Stroke; this relation will provide a concrete basis to determine the actuator dimensions for any application by picking up the operational force and stroke range. Furthermore, Figure 12 shows the possibility of enhancing the actuators curve by reducing the passive ends which is the main reason of the performance decline.

The simulation results show an excellent correlation with the results conducted by Danfoss PolyPower Benslimane et al. (2010). The behavioral outputs of the DEAP actuator has been simulated and sketched in Figure 11 and Figure 12. The outputs are determined using the introduced parameters shown in Table 5 and the governing equations introduced in section 4.2.

\section{Conclusion}

This work has addressed the analysis, modeling and simulation of multi-disciplinary systems using the Bond Graph. Through this work, the Bond Graph technique is introduced, characterized and implemented into two case studies. In case study I, the Bond Graph is used to analyze and simulate a fourth order electrical system. State-space equations have been derived. Moreover, the equivalent mechanical system is designed by conversion of the Bond Graph of the electrical circuit to mechanical system. The 20-Sim software has been employed in both case studies, whereby it is used in case study I for simulating and verifying the equality of the systems. The response of both electrical and mechanical systems were tested and verified by the simulation software. In case study II, to our knowledge for the first time, the modeling in the area of dielectric smart material using the Bond Graph method is investigated and highlighted as an original work. The output of the Bond Graph model enables finding the steady-state Voltage-Effective Stroke, Voltage-Effective Force and last but not least the Effective Force-Effective Stroke DEAP's push actuator characteristics. Throughout the modeling of the actuator, standard and verified dimensions and material parameters have been used for finding the actuator characteristics. Moreover, data is collected from practical experimentations and used to verify the Bond Graph model which concludes accurate correlation. 


\section{Acknowledgment}

The work was carried out at the premises of the University of Agder, Grimstad. At the University of Agder, appreciative acknowledgments to Professor Emeritus Hallvard Engja for his introductory course about the Bond Graph as well as for many discussions and beneficial advices about modeling multi-disciplinary systems.

\section{References}

Ashley, S. Artificial muscles. Scientific American, 2003. URL www.scientificamerican.com/ article.cfm?id=artificial-muscles.

Benslimane, M., Gravesen, P., and Sommer-Larsen, P. Mechanical properties of dielectric elastomers with smart metallic compliant electrodes. In Proc. of SPIE Int Soc. Opt. Eng. pages 150-157, 2002.

Benslimane, M., Kiil, H., and Tryson, M. Dielectric electro-active polymer push actuators: performance and challenges. Polymer International, 2010. $59(3): 415-421$.

Gawthrop, P. and Bevan, G. Bond-graph modeling. IEEE Control Systems Magazine, 2007. 27:24-45. doi:10.1109/MCS.2007.338279.

Iskandarani, Y. Mechanical energy harvesting of dielectric electrical activated polymers. Thesis Report, University of Southern Denmark, 2008.

Khurshid, A. and Malik, M. A. Bond graph modeling and simulation of impact dynamics of a car crash. In Intl. Bhurban Conf. on Applied Sciences $\&$ Technology, IBCAST. pages 63-67, 2007. doi:10.1109/INMIC.2003.1416738.

Pedersen, E. and Engja, H. Mathematical modeling and simulation of physical system. Lecture Notes, NTNU, Trondheim, 2001.

Pelrine, R., Kornbluh, R., Eckerle, J., Jeuck, P., Oh, S., Pei, Q., and Stanford, S. Dielectric elastomers: generators mode fundamentals and its applications. In Proc. SPIE conference on Electroactive Power Actuators and Devices. San Diego, pages 148-156, 2001.

Roman, M., Selisteanu, D., Bobasu, E., and Sendrescu, D. Bond graph modeling of a baker's yeast bioprocess. In Intl. Conf. on Modelling, Identification and Control (ICMIC). pages 82-87, 2010.

Wacker Chemie AG. Elastosil RT. In Datasheet 625. 2011. URL www. wacker.com.
Wissler, M., Mazza, E., and Kovacs, G. Electromechanical coupling in cylindrical dielectric elastomer actuators. Proc. SPIE 6524, 652409, 2007. doi:10.1117/12.714946.

Wong, Y. and Rad, A. Bond graph simulations of electrical systems. In Energy Management and Power Delivery. Proceedings of EMPD '98. pages 133-138, 1998. doi:10.1109/EMPD.1998.705489. 